\title{
Zeroes of Gaussian analytic functions
}

\author{
Mikhail Sodin * \\ School of Mathematics \\ Tel Aviv University \\ Tel Aviv 69978 Israel \\ sodin@post.tau.ac.il
}

February 1, 2008

\begin{abstract}
Geometrically, zeroes of a Gaussian analytic function are intersection points of an analytic curve in a Hilbert space with a randomly chosen hyperplane. Mathematical physics provides another interpretation as a gas of interacting particles. In the last decade, these interpretations influenced progress in understanding statistical patterns in the zeroes of Gaussian analytic functions, and led to the discovery of canonical models with invariant zero distribution. We shall discuss some of recent results in this area and mention several open questions.
\end{abstract}

\section{Introduction}

A Gaussian analytic function is a linear combination

$$
f(z)=\sum_{k \geq 0} \zeta_{k} f_{k}(z)
$$

of analytic functions $f_{k}: G \rightarrow \mathbb{C}(G \subseteq \mathbb{C}$ is a domain $)$,

$$
\sum_{k \geq 0}\left|f_{k}(z)\right|^{2}<\infty \quad \text { locally uniformly in } G
$$

with independent standard complex Gaussian random coefficients $\zeta_{k}$. The random zero set $\mathcal{Z}_{f}=f^{-1}(0)$ is the theme of this talk.

${ }^{*}$ Supported by the Israel Science Foundation of the Israel Academy of Sciences and Humanities. 
Pioneering contributions in this area were made by Paley and Wiener 28 , Chapter X], Kac [15. Chapter I], and Rice [30. Paley and Wiener constructed a large class of Gaussian analytic functions in a strip with stationary distribution with respect to the shifts, and computed the mean number of zeroes. Their work was influenced by ergodic theory and theory of almostperiodic functions. Kac was interested in the mean number of real zeroes of polynomials with real coefficients. Rice systematically treated both theoretical and applied aspects of random noises in radio signals. The technique introduced by Kac and Rice has been significant to radio engineers and physicists.

These studies were continued in various directions, notably by Littlewood and Offord [20, Hammersley [12, Offord [26, 27], and Kahane [16. Chapter 13]. Hammersley looked at pure probabilistic aspects of point processes generated by zeroes of random polynomials. The other authors were motivated by the entire functions and Nevanlinna theory. Introducing randomness, they tried to single out 'typical patterns' in the zero distribution in various classes of entire and analytic functions.

In the 90s, the subject was revived by several groups of researchers who came from different areas: Bogomolny, Bohigas and Leboeuf; Shub and Smale; Edelman and Kostlan; Hannay; Bleher, Shiffman and Zelditch; Nonnenmacher and Voros; by no means is this list complete. They established new links to physics (Coulomb gas of charged particles, random matrices, quantum chaos) and geometry (analytic curves in projective Hilbert spaces), and drastically changed the whole subject.

We start this lecture with a quick review of basic results pertaining to zero sets of arbitrary Gaussian analytic functions (Section 11). In Section 2 we introduce three canonical random zero process (on the Riemann sphere, complex plane, and the unit disc), distinguished by stationarity with respect to the isometries. In Sections $[3$ and 4 , we consider in more detail one of them, the canonical random zero process in $\mathbb{C}$. The exposition in this part is based on joint works with Tsirelson [34. In Section [5] we discuss an 'exactly solvable case' of the hyperbolic zero process recently discovered by Peres and Virág [29].

\section{The random zero process $\mathcal{Z}_{f}$}

Informally speaking, the random zero set $\mathcal{Z}_{f}=f^{-1}(0)$ is the intersection of an analytic curve $\mathfrak{f}: G \rightarrow P(H)$ in a projective Hilbert space with a random hyperplane, the analytic functions $f_{k}$ are homogeneous coordinates of the 
curve $\mathfrak{f}$. Projective unitary transformations of the curve $\mathfrak{f}$ do not change the random zero set $\mathcal{Z}_{f}$. Hence the random set $\mathcal{Z}_{f}$ depends only on geometry of the curve $\mathfrak{f}$. Its study can be interpreted as part of the H. Cartan - Ahlfors - H. and J. Weyl theory of analytic curves independent of the dimension of the target space. ${ }^{1}$

The properties of the random process $\mathcal{Z}_{f}$ can be expressed by its counting measure

$$
n_{f}=\sum_{a: f(a)=0} \delta_{a}
$$

$\delta_{a}$ is a point measure at $a$. The measure $n_{f}$ is a random, positive, locally finite measure on $G$. The classical formula

$$
n_{f}=\frac{1}{2 \pi} \Delta \log |f|
$$

(the Laplacian is understood in the sense of distributions) explicitly relates the random measure $n_{f}$ to the Gaussian analytic process $f$. Proofs of most of the results presented below start with this relation.

\subsection{The Edelman-Kostlan formula for the mean measure}

The first question about the random measure $n_{f}$ is to find its average which is a non-negative measure in $G$.

Theorem 1.2 (Edelman-Kostlan [9])

$$
\mathbb{E} n_{f}=\frac{1}{2 \pi} \Delta \log \|\mathfrak{f}\|
$$

where

$$
\|\mathfrak{f}\|(z):=\sqrt{\sum_{k \geq 0}\left|f_{k}(z)\right|^{2}} .
$$

I.e., the mean measure $\mathbb{E} n_{f}$ coincides with the Riesz measure of the subharmonic function $\log \|\mathfrak{f}\|(z)$.

The RHS of the Edelman-Kostlan formula is a pull-back of the FubiniStudy area measure from the projective space $P(H)$ to $G$ by the curve $\mathfrak{f}$. Its density with respect to the Euclidean area measure in $G$ equals

$$
\frac{1}{\pi}\left(\mathfrak{f}^{\#}\right)^{2}:=\frac{1}{\pi} \frac{\sum_{i<k}\left|f_{i}^{\prime} f_{k}-f_{i} f_{k}^{\prime}\right|^{2}}{\|\mathfrak{f}\|^{4}} .
$$

\footnotetext{
${ }^{1}$ An interesting attempt to build a 'dimensionless theory' of analytic curves was made by Favorov [10]. His approach is based on the pluripotential theory in Banach spaces.
} 
The function $\mathfrak{f}^{\#}$ is a 'Fubini-Study derivative' of the curve $\mathfrak{f}$. The EdelmanKostlan formula can be viewed as a version of the classical Crofton formula from the integral geometry. Its proof is a simple computation based on equation (1.1):

$$
\mathbb{E} n_{f}=\frac{1}{2 \pi} \Delta(\mathbb{E} \log |f|)=\frac{1}{2 \pi} \Delta \log \|f\|,
$$

since, for any complex Gaussian random variable $\zeta$,

$$
\mathbb{E} \log |\zeta|=\log \|\zeta\|+\text { const } .
$$

What about the higher 'moments' of the random measure $n_{f}$ ? They are expressed by the $k$-point correlation measures

$$
d \mu\left(z_{1}, \ldots z_{k}\right)=\mathbb{E}\left(d n_{f}\left(z_{1}\right) \ldots d n_{f}\left(z_{k}\right)\right)
$$

on $\underbrace{G \times \ldots G}_{k \text { times }}$. Hannay [13] derived explicit formulas for these measures which generalize (1.3). They involve determinants and permanents of $k \times k$ matrices. In different contexts, the rigorous proof of these formulas is given in [4] and [29].

\subsection{Calabi's rigidity}

Surprisingly, the mean $\mathbb{E} n_{f}$ determines the random zero set $\mathcal{Z}_{f}$. In geometry, the same phenomenon was discovered by Calabi already in the beginning of the $50 \mathrm{~s}$.

Theorem 1.5 Let $f$ and $g$ be Gaussian analytic functions in a domain $G$, and let $\mathbb{E} n_{f}=\mathbb{E} n_{g}$. Then the corresponding random zero sets $\mathcal{Z}_{f}$ and $\mathcal{Z}_{g}$ have the same distribution.

This holds due to the underlying analyticity. The idea is not difficult: let $K\left(z_{1}, z_{2}\right)=\mathbb{E}\left(f\left(z_{1}\right) \overline{f\left(z_{2}\right)}\right)$ be the covariance of the process $f$. By the Edelman-Kostlan formula, the mean measure $\mathbb{E} n_{f}$ determines the function $z \mapsto \log K(z, z)$ up to a harmonic summand. In turn, the diagonal $K(z, z)$ determines the whole covariance kernel $K$ (due to analyticity of $K$ in $z_{1}$ and $\overline{z_{2}}$ ), and hence the distribution of the Gaussian process $f$. The details and references are in 33 .

Here is Calabi's original formulation: If two linearly non-degenerate analytic curves $\mathfrak{f}: M \rightarrow P\left(H_{1}\right), \mathfrak{g}: M \rightarrow P\left(H_{2}\right)$ of a complex manifold $M$ induce the same Riemannian metric on $M$ by pulling back the corresponding Fubini-Study metrics, then the projective spaces coincide $P\left(H_{1}\right)=P\left(H_{2}\right)$, and the curves are unitarily equivalent. 


\subsection{Offord-type estimate}

Theorem 1.6 Let $f$ be a Gaussian analytic function on a plane domain $G$. Then for any test function $\phi \in C_{0}^{2}(G)$ with a compact support in $G$, and any $\lambda>0$

$$
\mathbb{P}\left(\left|\int_{G} \phi\left(d n_{f}-\mathbb{E}\left(d n_{f}\right)\right)\right|>\lambda\right) \leq 3 \exp \left(-\frac{2 \pi \lambda}{\|\Delta \phi\|_{1}}\right) .
$$

Here, $\|\cdot\|_{1}$ is the $L^{1}$ norm with respect to the area measure.

Here is an argument borrowed from Offord [26]. By (1.1) and Green's formula, we need to estimate the probability

$$
\mathbb{P}\left(\left|\int_{G}(\log |f|-\mathbb{E} \log |f|) \Delta \phi d m\right|>2 \pi \lambda\right),
$$

$m$ is the area measure. This reduces the proof to a simple fact about concentration of $\log |\zeta|$, where $\zeta$ is a complex Gaussian random variable. The details are in 33 .

The result can be extended in various directions. It persists for zero sets of any random analytic process $f$ in $G$ with uniformly bounded exponential moment: for some $c>0$,

$$
\sup _{z \in G} \mathbb{E}\left(e^{c|\log | f(z)||}\right)<\infty
$$

Examples of such analytic processes are given in 24]. Instead of the $L^{1}$ norm of the Laplacian $\Delta \phi$, one can fix any of the $L^{q}$-norms, $2<q \leq \infty$, of the gradient $\nabla \phi$. In Section 4, we discuss a more complicated 'global version' of Theorem 1.6.

There is a price for such a level of generality: sometimes, Offord's estimate does not give an optimal result. For example, it does not yield sharp bounds for the 'hole probability' (see (3.4) and (5.3) below).

\section{Chaotic analytic zero points}

In the beginning of the nineties, Bogomolny, Bohigas and Leboeuf; Kostlan; and Shub and Smale introduced a remarkably unique class of Gaussian analytic functions with unitary invariance of zero points. Following Hannay 13, we use the term 'chaotic analytic zero points' (CAZP, for short). We consider here three CAZP models: the elliptic CAZP, the flat CAZP, and 
the hyperbolic CAZP ${ }^{2}$. They are the random zero set of a Gaussian analytic function

$$
\begin{array}{ll}
f_{L}(z)=\sum_{k=0}^{L} \zeta_{k} \sqrt{\frac{L(L-1) \ldots(L-k+1)}{k !}} z^{k} & \text { (elliptic, } L=1,2, \ldots), \\
f_{L}(z)=\sum_{k=0}^{\infty} \zeta_{k} \sqrt{\frac{L^{k}}{k !} z^{k}} & \text { (flat, } L>0), \\
f_{L}(z)=\sum_{k=0}^{\infty} \zeta_{k} \sqrt{\frac{L(L+1) \ldots(L+k-1)}{k !}} z^{k} & \text { (hyperbolic, } L>0) .
\end{array}
$$

The analytic function (2.1) is a polynomial of degree $L$ (the domain of the elliptic CAZP is the Riemann sphere), the function (2.2) with probability one is an entire function, and the function (2.3) with probability one is analytic in the unit disc.

We introduce unified notation: $\mathcal{M}$ for the domain of the CAZP, and $\Gamma$ for the symmetry group of $\mathcal{M}$. Then $C A Z P$ is a unique $\Gamma$-stationary random zero process. Here, $\Gamma$-stationarity means that for any $\gamma \in \Gamma$ the point processes $\mathcal{Z}_{f}$ and $\mathcal{Z}_{f \circ \gamma}$ have the same distribution. Uniqueness means that CAZP is the only $\Gamma$-stationary process on $\mathcal{M}$ among the random zero sets of Gaussian analytic functions.

Having explicit formulas (2.1), (2.2), (2.3), it is very easy to prove $\Gamma$ stationarity and uniqueness. It suffices only to check that

$$
\mathbb{E} n_{f_{L}}=L \cdot m^{*}
$$

where $m^{*}$ is a normalized $\Gamma$-invariant area measure on $\mathcal{M}$. Then, by Calabi's rigidity, $\mathcal{Z}_{f_{L}}$ is $\Gamma$-stationary, and, again by Calabi's rigidity, $f_{L}$ is unique. Verification of (2.4) is a straightforward application of the Edelman-Kostlan formula. For example, in the flat case, $\left\|f_{L}\right\|^{2}(z)=\exp \left(L|z|^{2}\right)$, and

$$
n_{f_{L}}=\frac{1}{2 \pi} \Delta\left(L|z|^{2} / 2\right)=L \cdot \frac{1}{\pi} m .
$$

We see that in the flat case the normalized area $m^{*}$ equals $\frac{1}{\pi} m$. It is also worth mentioning that canonical isometric embeddings of $\mathcal{M}$ into projective Hilbert spaces corresponding to the Gaussian analytic functions (2.1), (2.2) and (2.3) are well known in geometry and physics.

\footnotetext{
${ }^{2}$ The toric CAZP (which we do not discuss here) was introduced by Nonnenmacher and Voros 25 .
} 
By (2.4), the parameter $L$ equals the mean number of random zeroes per unit area on $\mathcal{M}$. In what follows, we shall consider the asymptotic behaviour of the random zero processes $\mathcal{Z}_{f_{L}}$ in the 'large intensity limit' $L \rightarrow \infty$. Some features for all three canonical models are similar, some are different. Due to compactness, the elliptic model sometimes is simpler to analyze. On the other hand, the hyperbolic model has additional intriguing features.

To fix ideas, we shall concentrate on the flat model. In this case, introducing $L$, we just make a homothety of the plane with coefficient $r=\sqrt{L}$. This makes the flat case more transparent ${ }^{3}$. Thus, we do not need parameter $L$ anymore, and we consider the asymptotic zero distribution of the Gaussian entire function of order two

$$
f(z)=\sum_{k=0}^{\infty} \zeta_{k} \frac{z^{k}}{\sqrt{k !}} .
$$

The function $f(z)$ can be viewed as a Gaussian counterpart of the Weierstrass $\sigma$-function

$$
\sigma(z)=z \prod_{\omega \in \sqrt{\pi} \mathbb{Z}^{2} \backslash\{0\}}\left(1-\frac{z}{\omega}\right) e^{z / \omega+1 / 2(z / \omega)^{2}} .
$$

Indeed, the random function $|f(z)| e^{-|z|^{2} / 2}$ has a stationary distribution, while the function $|\sigma(z)| e^{-|z|^{2} / 2}$ has periods $\sqrt{\pi}$ and $i \sqrt{\pi}$.

\section{$3 \quad$ Linear statistics}

Given a test-function $h: \mathbb{C} \rightarrow \mathbb{R}$ with a compact support, consider the random variable

$$
Z_{r}(h)=\int h\left(\frac{z}{r}\right) d n(z), \quad \mathbb{E} Z_{r}(h)=\frac{r^{2}}{\pi} \int h d m,
$$

$n$ is a counting measure of the flat CAZP with intensity $L=1, m$ is the area measure. We are interested in the asymptotic behaviour of $Z_{r}(h)$ when $r \rightarrow \infty$. The size of fluctuations of $Z_{r}(h)$ depends on the smoothness of the test-function $h$.

\footnotetext{
${ }^{3}$ The scaling $z=z_{0}+\frac{w}{\sqrt{L}}$ flattens out the elliptic and hyperbolic geometry as $L \rightarrow \infty$. In this limit, the entire function (2.2) is a locally uniform limit of the functions (2.1) and (2.3), and the flat CAZP appears as a scaling limit of the other two CAZP models. This is the motivation for an advanced theory developed by Bleher, Shiffman and Zelditch in 4.
} 


\subsection{Smooth linear statistics}

Theorem 3.1 ([34]) Let $h$ be a $C^{2}$-function on $\mathbb{C}$ with a compact support. Then

$$
\operatorname{Var} Z_{r}(h)=\frac{\kappa+o(1)}{r^{2}}\|\Delta h\|_{2}^{2}, \quad r \rightarrow \infty,
$$

where $\kappa$ is a positive numerical constant. The random variables

$$
\frac{r}{\sqrt{\kappa}\|\Delta h\|_{2}}\left(Z_{r}(h)-\frac{r^{2}}{\pi} \int h d m\right)
$$

converge in distribution to the standard Gaussian law $\mathcal{N}(0 ; 1)$ for $r \rightarrow \infty$.

Asymptotic formula (3.2) first appeared in Forrester and Honner [1]. It is worth mentioning that Theorem 3.1 persists for the other two CAZP models in the large intensity limit $L \rightarrow \infty$ [34, Part I].

It is instructive to compare (3.2) with the size of variations for a simple point process in the plane given by i.i.d. Gaussian perturbations of the lattice. Consider the point process

$$
\mathcal{S}=\left\{\sqrt{\pi}(k+i l)+\eta_{k, l}:(k, l) \in \mathbb{Z}^{2}\right\}
$$

where $\eta_{k, l}$ are independent standard complex Gaussian random variables. In this case, $\operatorname{Var} S_{r}(h) \sim$ const $\|\nabla h\|_{2}^{2}$, for $r \rightarrow \infty$. This is rather different from (3.2). Asymptotic similarity to the flat CAZP $\mathcal{Z}_{f}$ can be achieved by inventing special correlations between the perturbations $\eta_{k, l} .{ }^{4}$ In Section 4 we return to the idea of the flat CAZP as a perturbed lattice.

The proof of Theorem 3.1 starts with Green formula

$$
Z_{r}(h)-\frac{r^{2}}{\pi} \int h d m=\frac{1}{2 \pi} \int \log \left|f_{r}^{*}\right| \Delta h d m, \quad f_{r}^{*}(z)=\frac{f(r z)}{\sqrt{\operatorname{Var} f(r z)}} .
$$

The RHS is a non-linear functional on a Gaussian process $f_{r}^{*}$. The rest is based on the method of moments á la Breuer and Major [5]: we expand the function $\zeta \mapsto \log |\zeta|$ in Hermite polynomials in the space $L_{\mathbb{C}}^{2}\left(e^{-|\zeta|^{2}}\right)$ (the Wick expansion), and evaluate the moments of $Z_{r}(h)$ using the combinatorial diagram technique.

\footnotetext{
${ }^{4}$ Lattice points are aggregated into clusters and each cluster scatters in a special (equiangular and equidistant) way 34 Part I, Introduction].
} 


\subsection{Number of chaotic analytic zero points}

Let $\Omega \subset \mathbb{C}$ be a bounded domain with a piecewise smooth boundary. We are interested in the asymptotic behaviour of the random variable $n(r \Omega)=$ $Z_{r}\left(\mathbb{1}_{\Omega}\right)$ for $r \rightarrow \infty$. Forrester and Honner [1] argued that the technique developed by Martin and Yalçin 22 for studying the Gibbs states of infinite systems of charged particles applied to the flat CAZP gives

$$
\operatorname{Var} n(r \Omega)=r \cdot(\tau+o(1)) \text { Length }(\partial \Omega), \quad r \rightarrow \infty,
$$

$\tau$ is a positive numerical constant. This is consistent with the idea that the variation of the number of points in $r \Omega$ should behave like the number of points in the 'strip' of constant size around the boundary $\partial(r \Omega)$.

Presumably, the method of Martin and Yalçin also yields that the random variables

$$
\frac{n(r \Omega)-\pi^{-1} r^{2} m(\Omega)}{\sqrt{r \cdot \tau \operatorname{Length}(\partial \Omega)}}
$$

converge in distribution to $\mathcal{N}(0 ; 1)$ for $r \rightarrow \infty$.

It would be interesting to find a counterpart of the law of the iterated logarithm; i.e. to find a function $\phi(r)$ such that with probability one

$$
\limsup _{r \rightarrow \infty} \frac{\left|n(r)-r^{2}\right|}{\sqrt{r} \phi(r)}=1 .
$$

Here $n(r)=n(\{|z| \leq r\})$.

\subsection{The 'hole probability' and large deviations}

The next theorem proves an estimate conjectured by Yuval Peres:

Theorem $3.3([34])$ For $r \geq 1$,

$$
e^{-c_{1} r^{4}} \leq \mathbb{P}(n(r)=0) \leq e^{-c_{2} r^{4}},
$$

where $n(r)=n(\{|z| \leq r\})$, and $c_{1}$ and $c_{2}$ are positive numerical constants.

It would be interesting to check whether there exists the limit

$$
\lim _{r \rightarrow \infty} \frac{\log ^{-} \mathbb{P}(n(r)=0)}{r^{4}}
$$

and (if it does) to compute its value.

The lower bound in (3.4) is obtained by an explicit construction. The upper bound follows from 
Theorem 3.5 ([34]) For any $\delta \in\left(0, \frac{1}{4}\right]$ and $r \geq 1$,

$$
\mathbb{P}\left(\left|\frac{n(r)}{r^{2}}-1\right| \geq \delta\right) \leq e^{-c(\delta) r^{4}} .
$$

The proof of Theorem 3.5 uses tools from the entire functions theory. First, we show that with very high probability $\log \max _{r \mathbb{D}}|f|$ is close to $r^{2} / 2$. Then, estimating $\log |f|$ from below, we show that with very high probability the average

$$
\int_{0}^{2 \pi} \log \left|f\left(r e^{i \theta}\right)\right| \frac{d \theta}{2 \pi}
$$

is also close to $r^{2} / 2$. From this, using Jensen's formula, we deduce Theorem 3.5

Theorems 3.3 and 3.5 are consistent with the results known for a one component Coulomb system of charged particles of one sign embedded into a uniform background of the opposite sign, Jancovici, Lebowitz and Magnificat [18. It would be good to understand the asymptotic behaviour of the random variable $r^{-\alpha}\left[n(r)-r^{2}\right]$ for $r \rightarrow \infty$ and $\alpha \geq 1 / 2$. At present, we understand the extreme case $\alpha=\frac{1}{2}$, and (not completely) the case $\alpha \geq 2$. A plausible guess (motivated by [18]) is

$$
\lim _{r \rightarrow \infty} \frac{\log \log \mathbb{P}\left(\left|n(r)-r^{2}\right| \geq r^{\alpha}\right)}{\log r}= \begin{cases}2 \alpha-1, & \frac{1}{2} \leq \alpha \leq 1 \\ 3 \alpha-2, & 1 \leq \alpha \leq 2 \\ 2 \alpha, & \alpha \geq 2\end{cases}
$$

In the first case, the normalized charge $\left|n(r)-\frac{r^{2}}{2}\right|$ grows slower than the perimeter of the disc, in the second case it grows faster than the perimeter but slower than the area, in the last case (so called 'overcrowding') it grows faster than the area. According to the philosophy of [18, this should lead to a change of the asymptotic regime at $\alpha=1$ and $\alpha=2$. The technique we developed for the proof of Theorem 3.3 helps to analyze the case $\alpha>2$. The other two cases seem to require a different technique.

\section{Flat chaotic analytic zero points as a perturbed lattice}

How evenly do the flat CAZP spread over the plane? In the euclidean case we have a very natural system of points spread evenly throughout the plane, namely, the lattice points. Instead of comparing the random counting 
measure $n_{f}$ with its average $\frac{1}{\pi} m$, we consider the flat CAZP as a perturbed lattice $\left\{\sqrt{\pi}(k+i l)+\xi_{k, l}: k, l \in \mathbb{Z}\right\}$, for some (dependent) complex-valued random variables $\xi_{k, l}$. We may hope for fast decay of the tail probabilities $\mathbb{P}\left(\left|\xi_{k, l}\right| \geq \lambda\right)$ for large $\lambda$, uniformly in $(k, l) \in \mathbb{Z}^{2}$. The uniformity becomes trivial if the distribution of $\left(\xi_{k, l}\right)$ is invariant under lattice shifts. We treat the random variables $\xi_{k, l}$ as measurable functions on the space $\Omega=\mathbb{C}^{\mathbb{Z}^{2}}$ of two-dimensional arrays $\xi: \mathbb{Z}^{2} \rightarrow \mathbb{C}$.

Theorem 4.1 ([34]) There exists a probability measure $\mathbb{P}$ on $\Omega$ such that (i) $\mathbb{P}$ is invariant under the shifts of $\mathbb{Z}^{2}$;

(ii) the random set $\mathcal{S}=\left\{\sqrt{\pi}(k+i l)+\xi_{k, l}:(k, l) \in \mathbb{Z}^{2}\right\}$ is distributed like the flat $C A Z P \mathcal{Z}$;

(iii) $\mathbb{E}\left(e^{\epsilon\left|\xi_{0,0}\right|^{2}}\right)<\infty$ for some $\epsilon>0$.

This result gives no information about correlation between $\xi_{k, l}$. Probably, they can be chosen to be nearly independent on large distances.

The proof of Theorem 4.1 does not use the Gaussian nature of the flat CAZP but only the uniform boundedness of the exponential moment of the 'random potential' $u(z)=\log |f(z)|-\frac{1}{2}|z|^{2}$. The main ingredients of the proof are the M. Hall's 'marriage lemma' (needed to match the flat CAZP with the lattice $\sqrt{\pi} \mathbb{Z}^{2}$ ), and a potential theory lemma which can be useful in other discrepancy problems:

Lemma 4.2 Let $u$ be a bounded delta-subharmonic function on $\mathbb{C}$ (i.e., a difference of two subharmonic functions), and let $\Delta u=\mu-m$ in the distributional sense, $\mu$ is a non-negative measure. Then for any bounded Borel set $E \subset \mathbb{C}$

$$
\mu(E) \leq m\left(E_{+t}\right) \quad \text { and } \quad m(E) \leq \mu\left(E_{+t}\right),
$$

where $E_{+t}=\{z: \operatorname{dist}(z, E) \leq t\}$ is a t-vicinity of $E, t=$ const $\|u\|_{\infty}^{1 / 2}$.

The boundedness of $u$ is too strong for applications. It can be easily weakened by convolving $u$ with a smooth convolutor supported by an appropriate disc.

The two ingredients described above alone help to prove only a local result in the spirit of Theorem 4.1. Globalization is still a problem: after smoothing, the random potential $u(z)=\log |f(z)|-\frac{1}{2}|z|^{2}$ is almost surely unbounded. Rare fluctuations appear somewhere on the infinite plane $\mathbb{C}$, though probably far from the origin. To achieve some locality, we introduce a special adaptive metric $\rho$ on $\mathbb{C}$ using Hörmander's construction [14, Section 1.4]. This metric $\rho$ is small where the potential is large. Then we use a 
version of Lemma 4.2 making use of $\rho$-neighbourhoods instead of euclidean ones.

It is worth mentioning that in [1] Ajtai, Komlós and Tusnády studied high probability matchings of a system of $N^{2}$ independent random points $\Lambda=\left\{\lambda_{1}, \ldots \lambda_{N^{2}}\right\}$ uniformly distributed in the square $[0, N]^{2} \subset \mathbb{R}^{2}$ with the grid $\left\{\omega_{1}, \ldots \omega_{N^{2}}\right\}=[0, N) \cap \mathbb{Z}^{2}$. They considered the average transportation

$$
T(\Lambda):=\min _{\pi} \frac{1}{N^{2}} \sum_{1 \leq i \leq N^{2}}\left|\lambda_{i}-\omega_{\pi(i)}\right|,
$$

the minimum is taken over all permutations $\pi$ of $\left\{1,2, \ldots N^{2}\right\}$. Then with high probability

$$
\text { const } \sqrt{\log N} \leq T(\Lambda) \leq \text { Const } \sqrt{\log N} .
$$

For related results see Leighton and Shor [19], and Talagrand [35. In our global set-up we deal with infinite measures in the plane. Then, according to the lower bound in (4.3), for any matching the average transportation distance tends to infinity in the $N \rightarrow \infty$ limit. This leaves no hope for the finite average distance matching between the Poissonian point process in $\mathbb{R}^{2}$ and a lattice, even without a quantitative estimate (iii).

It would be interesting to find a hyperbolic counterpart of Theorem 4.1

\section{Hyperbolic CAZP}

The hyperbolic CAZP, in contrast to the other models, depends on two intrinsic parameters: the mean density of zeroes per unit hyperbolic area, and the size of the sets on which we count the number of points. For instance, $n_{L}\left(D_{r}\right)$ is a number of the hyperbolic CAZP with intensity $L$ in the hyperbolic disc of radius $r$. This leads to different asymptotic regimes, and makes the hyperbolic model richer than the other two. It is also worth mentioning that, for different values of the intensity $L$, the Gaussian analytic function (2.3) exhibits completely different patterns in the asymptotic behaviour when $z$ approaches the boundary of the unit disc Kahane 16. Chapter 13].

An interesting observation by Diaconis and Evans [8], and Peres and Virág [29] says that the real part of the hyperbolic random function (2.3) up to a constant term is a Poisson integral of a Gaussian random noise on the unit circle. In the case $L=1$ this is classical white noise [29, p.11], the case $L=2$ corresponds to the Gaussian process on the Dirichlet space $H_{2}^{1 / 2}(\mathbb{T})$ [8, Example 5.6]. 


\subsection{An exactly solvable model}

Here, we discuss a recent finding of Peres and Virág [29] which pertains to the case $L=1$. Recall that the $k$-point correlation function $p\left(z_{1}, \ldots z_{k}\right)$ of a random point process is

$$
p\left(z_{1}, \ldots z_{k}\right)=\lim _{\epsilon \rightarrow 0} \frac{p_{\epsilon}\left(z_{1}, \ldots z_{k}\right)}{\left(\pi \epsilon^{2}\right)^{k}},
$$

where $p_{\epsilon}\left(z_{1}, \ldots z_{k}\right)$ is the probability that each $\operatorname{disc}\left\{\left|z-z_{j}\right| \leq \epsilon\right\}, 1 \leq$ $j \leq k$, contains at least one point of the process. For the random zero processes of a Gaussian analytic function, the limit on the RHS always exists. An equivalent definition says that $p\left(z_{1}, \ldots z_{k}\right)$ is a density of the $k$-point correlation measure (1.4) with respect to the Lebesgue measure. The correlation measure also contains singular terms supported by the large diagonal; these terms are expressed via $j$-point correlation functions with $j<k$. Thus, the random zero process can be described by its correlation functions.

Using Hannay's formulas [13, Peres and Virág proved

Theorem 5.1 (Peres-Virág [29]) The correlation function of the hyperbolic CAZP with intensity $L=1$ is

$$
p\left(z_{1}, \ldots z_{k}\right)=\pi^{-n} \operatorname{det}\left[\frac{1}{\left(1-z_{i} \bar{z}_{j}\right)^{2}}\right]_{1 \leq i, j \leq k} .
$$

This remarkable identity makes the hyperbolic CAZP with $L=1$ an 'exactly solvable model' among all CAZP. ${ }^{5}$ In particular, it yields amazingly simple explicit expressions for the distribution of the number of zeroes $n(\rho)$ in the disc $\{|z| \leq \rho\}$ and for the asymptotics of the 'hole probability':

Corollary $5.2([29])$ Let $\mathcal{Z}$ be the hyperbolic $C A Z P$ with intensity $L=1$. Then

(i) $n(\rho)$ has the same distribution as $\sum_{j=1}^{\infty} X_{j}$ where $\left\{X_{j}\right\}$ is a sequence of independent Bernoulli random variables with $\mathbb{P}\left(X_{j}=1\right)=\rho^{2 j}$;

(ii) for $\rho \rightarrow 1$

$$
\mathbb{P}(n(\rho)=0)=\exp \left[-\frac{\pi^{2}+o(1)}{1-\rho}\right]
$$

(iii) the ratio

$$
\frac{n(\rho)-\mathbb{E} n(\rho)}{\sqrt{\operatorname{Var} n(\rho)}}
$$

\footnotetext{
${ }^{5}$ Peres and Virág observe that this is the only determinantal process among CAZP.
} 
(with $\mathbb{E} n(\rho)=\frac{\rho^{2}}{1-\rho^{2}}$ and $\left.\operatorname{Var} n(\rho)=\frac{\rho^{2}}{1-\rho^{4}}\right)$ converges in distribution to the standard Gaussian law $\mathcal{N}(0 ; 1)$ for $\rho \rightarrow 1$.

In this case, Var $n(\rho)$ has the same order of magnitude as $\mathbb{E} n(\rho)$ whilst in the flat case the variance grows only as a square root of the mean. This naturally reflects the difference between the hyperbolic and euclidean geometries: in hyperbolic geometry the perimeter grows like the area, and much more random zeroes are located near the boundary circumference.

We are not aware of counterparts of (ii) and (iii) for the hyperbolic CAZP with $L \neq 1$.

\section{Loose ends}

In this lecture we've only touched the 'ground level' of the theory. There are plenty of interesting and deep developments. Among them are

- scaling limits of zeroes of random polynomials (Ibragimov and Zeitouni 17] and Shiffman and Zelditch 31 ) and of random holomorphic sections of high powers of Hermitian line bundles (Bleher, Shiffman and Zelditch [4]);

- solutions of random systems of algebraic equations, including sparse systems (see Edelman and Kostlan [9, Malajovich and Rojas 21], Shiffman and Zelditch 32 and references therein);

- distribution of real zeroes of random real polynomials (Maslova 23, Dembo, Poonen, Shao and Zeitouni [6], Bleher and Di [3], Aldous and Fyodorov [2]);

- links with the zero distribution of chaotic eigenfunctions (Nonnenmacher and Voros [25], Hannay [13]) and with the distribution of eigenvalues of random matrices with independent complex Gaussian entries (Forrester and Honner [11, Diaconis and Evans [8], Dennis and Hannay [7] and references therein),

and each of them deserves a special lecture (cf. [36]). But these are different stories to be told by other people.

Acknowledgement For the last four years, I have been enjoying collaboration with Boris Tsirelson on the subject of this lecture. I am grateful to him for his encouragement and patience. I am also grateful to Fëdor Nazarov, Leonid Pastur, Yuval Peres, Leonid Polterovich, Zeev Rudnick, Bernard Shiffman, Peter Yuditskii, and Steve Zelditch for useful discussions. 


\section{References}

[1] M. Ajtai, J. Komlós and G. Tusnády, On optimal matchings. Combinatorica 4 (1984), 259-264.

[2] A. Aldous and Y. Fyodorov, Real roots of random polynomials: universality close to accumulation points. J. Phys. A 37 (2004), 1231-1239.

[3] P. Bleher and Xiaojun Di, Correlations between zeros of non-Gaussian random polynomials. arXiv:math.MP/0308014

[4] P. Bleher, B. Shiffman and S. Zelditch, Universality and scaling of correlations between zeros on complex manifolds. Invent. Math. 142 (2000), 351-395; Poincaré-Lelong approach to universality and scaling of correlations between zeros. Comm. Math. Phys. 208 (2000), 771-785.

[5] P. Breuer and P. Major, Central limit theorems for nonlinear functionals of Gaussian fields. J. Multivariate Anal. 13 (1983), 425-441.

[6] A. Dembo, B. Poonen, Qi-Man Shao and O. Zeitouni. Random polynomials having few or no real zeros. J. Amer. Math. Soc. 15 (2002), 857-892.

[7] M. R. Dennis and J. H. Hannay, Saddle points in the chaotic analytic function and Ginibre characteristic polynomial. J. Phys. A 36 (2003), $3379-3383$.

[8] P. Diaconis and S. Evans, Linear functionals of eigenvalues of random matrices. Trans. Amer. Math. Soc. 353 (2001), 2615-2633.

[9] A. Edelman and E. Kostlan, How many zeros of a random polynomial are real? Bull. Amer. Math.Soc. (N.S.) 32 (1995), 1-37.

[10] S. Yu. Favorov, Growth and distribution of the values of holomorphic mappings of a finite-dimensional space into a Banach space. Siberian Math. J. 31 (1990), 137-146; On the growth of holomorphic mappings from a finite-dimensional space into a Banach space. Mat. Fiz. Anal. Geom. (Kharkov) 1 (1994), 240-251. (Russian)

[11] P. J. Forrester and G. Honner, Exact statistical properties of the zeros of complex random polynomials. J. Phys. A 32 (1999), 2961-2981.

[12] J. M. Hammersley, The zeros of a random polynomial. Proceedings of the Third Berkeley Symposium on Mathematical Statistics and Probability, 1954-1955, vol. II, pp. 89-111. 
[13] J. H. Hannay, Chaotic analytic zero points: exact statistics for those of a random spin state. J. Phys. A 29 (1996), L101-L105. The chaotic analytic function. J. Phys. A 31 (1998), L755-L761.

[14] L. Hörmander, The analysis of linear partial differential operators. I. Distribution theory and Fourier analysis. Grundlehren der Mathematischen Wissenschaften, 256. Springer-Verlag, Berlin, 1983.

[15] M. Kac, Probability and related topics in physical sciences. Lectures in Applied Mathematics. Proceedings of the Summer Seminar, Boulder, Colo., 1957, Vol. I Interscience Publishers, London-New York, 1959.

[16] J.-P. Kahane, Some random series of functions (2nd ed). Cambridge University Press, Cambridge, 1985.

[17] I. Ibragimov and O. Zeitouni, On roots of random polynomials, Trans. Amer. Math. Soc. 349 (1997), 2427-2441.

[18] B. Jancovici, J. L. Lebowitz and G. Magnificat, Large charge fluctuations in classical Coulomb systems. J. Statist. Phys. 72 (1993), 773-787.

[19] T. Leighton and P. Shor, Tight bounds for minimax grid matching with applications to the average case analysis of algorithms. Combinatorica 9 (1989), 161-187.

[20] J. E. Littlewood and A. C. Offord, On the distribution of zeros and $a$-values of a random integral function. II. Ann. of Math. (2) 49 (1948), 885-952; errata 50 (1949), 990-991.

[21] G. Malajovich and M. Rojas, Polynomial systems and the momentum map. Foundations of computational mathematics (Hong Kong, 2000), 251-266, World Sci. Publishing, River Edge, NJ, 2002.

[22] Ph. A. Martin and T. Yalçin, The charge fluctuations in classical Coulomb systems. J. Statist. Phys. 22 (1980), 435-463.

[23] N. B. Maslova, The variance of the number of real roots of random polynomials. Teor. Verojatnost. i Primenen. 19 (1974), 36-51. (Russian); The distribution of the number of real roots of random polynomials. Teor. Verojatnost. i Primenen. 19 (1974), 488-500. (Russian)

[24] F. Nazarov, M. Sodin and A. Volberg, The geometric Kannan-LovaszSimonovits lemma, dimension-free estimates for the distribution of the values of polynomials, and the distribution of the zeros of random analytic 
functions. St. Petersburg Math. J. 14 (2003), 351-366; Local dimensionfree estimates for volumes of sublevel sets of analytic functions. Israel J. Math. 133 (2003), 269-283.

[25] S. Nonnenmacher and A. Voros, Chaotic eigenfunctions in phase state. J. Stat. Phys. 92 (1998), 431-518.

[26] A. C. Offord, The distribution of zeros of power series whose coefficients are independent random variables. Indian J. Math. 9 (1967), 175-196.

[27] A. C. Offord, The distribution of the values of an entire function whose coefficients are independent random variables, I. Proc. London Math. Soc. (3) 14a (1965) 199-238; II. Math. Proc. Cambridge Philos. Soc. 118 (1995), 527-542;

[28] R. E. A. C. Paley and N. Wiener, Fourier transforms in the complex domain. AMS Colloquium Publications, 1934.

[29] Yu. Peres and B. Virág, Zeros of the i.i.d. Gaussian power series and a conformal invariant determinantal process. arXiv:math.PR/0310297

[30] S. O. Rice, Mathematical analysis of random noise. Bell System Tech. J. 23 (1944), 282-332; Ibid 24 (1945), 46-156.

[31] B. Shiffman and S. Zelditch, Equilibrium distribution of zeros of random polynomials. IMRN (2003) no. 1, 25-49.

[32] B. Shiffman and S. Zelditch, Random polynomials with prescribed Newton polytope. J. Amer. Math. Soc. 17 (2004), 49-108.

[33] M. Sodin, Zeroes of Gaussian analytic functions. Math. Res. Lett. 7 (2000), 371-381.

[34] M. Sodin and B. Tsirelson, Random complex zeroes. I Asympotic normality arXiv:math.CV/0210090, Israel Journ. Math., to appear; II. Perturbed lattice. arXiv:math.CV/0309449, III. Decay of the hole probability. arXiv:math.CV/0312258.

[35] M. Talagrand, Matching theorems and empirical discrepancy computations using majorizing measures. J. Amer. Math. Soc. 7 (1994), 455-537.

[36] S. Zelditch, ¿From random polynomials to symplectic geometry. XIIIth International Congress on Mathematical Physics (London, 2000), 367376, Int. Press, Boston, MA, 2001; Asymptotics of polynomials and eigen- 
functions. Proceedings of the International Congress of Mathematicians, Vol. II (Beijing, 2002), 733-742, Higher Ed. Press, Beijing, 2002. 